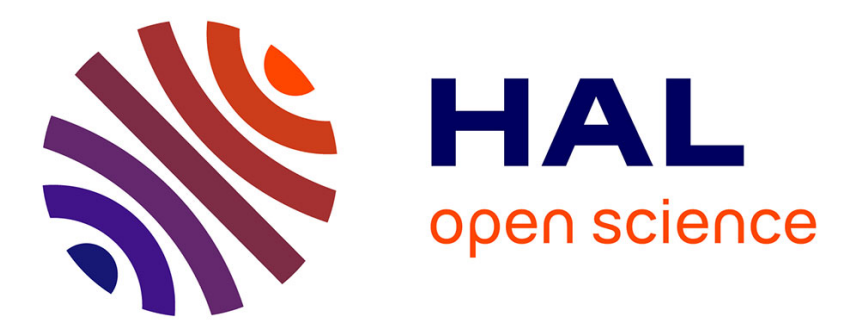

\title{
Baldovin-Stella stochastic volatility process and Wiener process mixtures
}

Pier Paolo Peirano, Damien Challet

\section{To cite this version:}

Pier Paolo Peirano, Damien Challet. Baldovin-Stella stochastic volatility process and Wiener process mixtures. The European Physical Journal B: Condensed Matter and Complex Systems, 2012, 85 (8), pp.276. 10.1140/epjb/e2012-30134-y . hal-00734355

\section{HAL Id: hal-00734355 \\ https://hal.science/hal-00734355}

Submitted on 21 Sep 2012

HAL is a multi-disciplinary open access archive for the deposit and dissemination of scientific research documents, whether they are published or not. The documents may come from teaching and research institutions in France or abroad, or from public or private research centers.
L'archive ouverte pluridisciplinaire HAL, est destinée au dépôt et à la diffusion de documents scientifiques de niveau recherche, publiés ou non, émanant des établissements d'enseignement et de recherche français ou étrangers, des laboratoires publics ou privés. 


\title{
Baldovin-Stella stochastic volatility process and Wiener
}

\section{Process Mixtures}

Pier Paolo Peirano ${ }^{1}$ and Damien Challet $^{2,3}$

1 Capital Fund Management, 6-8 boulevard Hausmann, 75009 Paris, France, pier-paolo.peirano@cfm.fr

${ }^{2}$ Laboratoire de mathmatiques appliques aux systmes, cole Centrale Paris, Grande Voie des Vignes, 92295 Chtenay-Malabry, France, damien.challet@ecp.fr

${ }^{3}$ Encelade Capital SA, ch. du Bochet 8a, 1025 St-Sulpice, Switzerland

Received: date / Revised version: date

\begin{abstract}
Starting from inhomogeneous time scaling and linear decorrelation between successive price returns, Baldovin and Stella recently proposed a powerful and consistent way to build a model describing the time evolution of a financial index. We first make it fully explicit by using Student distributions instead of power law-truncated Lévy distributions and show that the analytic tractability of the model extends to the larger class of symmetric generalized hyperbolic distributions and provide a full computation of their multivariate characteristic functions; more generally, we show that the stochastic processes arising in this framework are representable as mixtures of Wiener processes. The basic Baldovin and Stella model, while mimicking well volatility relaxation phenomena such as the Omori law, fails to reproduce other stylized facts such as the leverage effect or some time reversal asymmetries. We discuss how to modify the dynamics of this process in order to reproduce real data more accurately.
\end{abstract}

\section{How Scaling and Efficiency Constrains}

\section{Return Distribution}

Finding a faithful stochastic model of price time series is still an open problem. Not only should it replicate in a unified way all the empirical statistical regularities, often called stylized facts, (cf e.g. [1,2]), but it should also be easy to calibrate and analytically tractable, so as to facilitate its application to derivative pricing and financial risk assessment. Up to now none of the proposed models has been able to meet all these requirements despite their variety. Attempts include ARCH family ([3,4] and references therein), stochastic volatility ([5] and references therein), multifractal models ([6-9] and references therein), multi- 
timescale models ([10-12]), Lévy processes ([13] and references therein), and self-similar processes ([14]).

Recently Baldovin and Stella (Ba-St thereafter) proposed a new way of addressing the question. We advise the reader to refer to the original papers [15-17] for a full description of the model as we shall only give a brief account of its main underlying principles. Using their notation let $S(t)$ be the value of the asset under consideration at time $t$, the logarithmic return over the interval $[t, t+\delta t]$ is given by $r_{t, \delta t}=\ln S(t+\delta t)-\ln S(t)$; the elementary time unit is a day, i.e., $t=0,1, \ldots$ and $\delta t=1,2, \ldots$ days. In order to accommodate for non-stationary features, the distribution of $r_{t, \delta t}$ is denoted by $P_{t, \delta t}(r)$ which contains an explicit dependence on $t$. The most impressive achievement of Ba-St is to build the multivariate distribution $P_{0,1}^{(n)}\left(r_{0,1}, \ldots, r_{n, 1}\right)$ of $n$ consecutive daily returns starting from the univariate distribution of a single day provided that the following conditions hold:

1. No trivial arbitrage: the returns are linearly independent, i.e. $E\left(r_{i, 1}, r_{j, 1}\right)=0$ for $i \neq j$, with the standard condition $E\left(r_{i, 1}\right)=0$.

2. Possibly anomalous scaling of the return distribution with respect to the time interval $\delta t$, with exponent $D:^{1}$

$$
P_{0, \delta t}(r)=\frac{1}{\delta t^{D}} P_{0,1}\left(\frac{r}{\delta t^{D}}\right)
$$

3. Identical form of the unconditional distributions of the daily returns up to a possible dependence of the vari-

\footnotetext{
1 See Ref. [18] for a recent review of anomalous scaling in
} finance. ance on the time $t$, i.e.

$$
P_{t, 1}(r)=\frac{1}{a_{t}} P_{0,1}\left(\frac{r}{a_{t}}\right) .
$$

As shown in the addendum of [16] these conditions admit the solution

$$
f_{0,1}^{(n)}\left(k_{1}, \ldots, k_{n}\right)=\tilde{g}\left(\sqrt{a_{1}^{2 D} k_{1}^{2}+\cdots+a_{n}^{2 D} k_{n}^{2}}\right),
$$

where $f_{0,1}^{(n)}$ is the characteristic function of $P_{0,1}^{(n)}, \tilde{g}$ the characteristic function of $P_{0,1}$, and $a_{i}^{2 D}=i^{2 D}-(i-1)^{2 D}$. In this way the full process is entirely determined by the choice of the scaling exponent $D$ and the distribution $P_{0,1}$.

Therefore the characteristic function of $P_{t, \delta t}(r)$ is

$$
\begin{aligned}
f_{t, T}(k) & =f_{0,1}^{(n)}(\underbrace{0, \ldots, 0}_{t \text { terms }}, \underbrace{k, \ldots, k}_{\delta t \text { terms }}, 0, \ldots, 0) \\
& =\tilde{g}\left(k \sqrt{(t+\delta t)^{2 D}-t^{2 D}}\right),
\end{aligned}
$$

i.e.

$P_{t, \delta t}(r)=\frac{1}{\sqrt{(t+\delta t)^{2 D}-t^{2 D}}} P_{0,1}\left(\frac{r}{\sqrt{(t+\delta t)^{2 D}-t^{2 D}}}\right)$.

The functional form of $\tilde{g}$ in Eq. (1) introduces a dependence between the unconditional marginal distributions of the daily returns by the means of a generalized multiplication $\otimes$ in the space of characteristic functions, i.e.,

$$
f_{0,1}^{(n)}\left(k_{1}, \ldots, k_{n}\right)=\tilde{g}\left(a_{1}^{D} k_{1}\right) \otimes_{\tilde{g}} \cdots \otimes_{\tilde{g}} \tilde{g}\left(a_{n}^{D} k_{n}\right),
$$

with $\otimes_{\tilde{g}}$ defined by

$$
x \otimes_{\tilde{g}} y=\tilde{g}\left(\sqrt{\left[\tilde{g}^{-1}(x)\right]^{2}+\left[\tilde{g}^{-1}(y)\right]^{2}}\right) .
$$

At first sight this last equation may seem a trivial identity, but it does hide a powerful statement. Suppose indeed that instead of starting with the probability distribution $\tilde{g}$, one 
takes a general distribution with finite variance $\sigma^{2}=2$ and characteristic function $\tilde{p}_{1}$, then it is shown in [15] that

$$
\lim _{N \rightarrow \infty} \underbrace{\tilde{p}_{1}\left(\frac{k}{\sqrt{N}}\right) \otimes_{\tilde{g}} \cdots \otimes_{\tilde{g}} \tilde{p}_{1}\left(\frac{k}{\sqrt{N}}\right)}_{N \text { terms }}=\tilde{g}(k) .
$$

This means that in this framework the return distribution at large scales is independent of the distribution of the returns at microscopic scales: it is completely determined by the correlation introduced by the multiplication $\otimes_{\tilde{g}}$, with fixed point $\tilde{g}$. Note that if $\tilde{g}$ is the characteristic function of the Gaussian distribution, then $\otimes_{\tilde{g}}$ reduces to the standard multiplication and one recovers the standard Central Theorem Limit.

As the volatility of the model shrinks in an inexorable way, Baldovin and Stella propose to restart the whole shrinking process after a critical time $\tau_{c}$ long enough for the volatility autocorrelation to fall to the noise level. In this way one recovers a sort of stationary time series when their length is much greater than $\tau_{c}$. In this case one expects that the empirical distribution of the return $\bar{P}_{\delta t}(r)$ over a time horizon $\delta t \ll \tau_{c}$, evaluated with a sliding window satisfies

$$
\bar{P}_{\delta t}(r)=\frac{1}{\tau_{c}} \sum_{t=0}^{\tau_{c}-1} P_{t, \delta t}(r) .
$$

In the original papers no market mechanism is proposed for modeling the restart of the process; it is simply stated that the length of different runs and the starting points of the processes could be stochastic variables. In their simulations the length of the processes was fixed to $\tau=500$, which corresponds to slightly more than two years of daily data. The current approach to restarts is less rigid. [19] propose to have Poisonnian restarts, which decouples more clearly the dynamics into endegeneous and exogeneous parts and allows for easier calibration.

\section{A Fully Explicit Theory with Student}

\section{Distributions}

In [16] a power law truncated Lévy distribution is chosen to describe the returns

$$
\tilde{g}(k)=\exp \left(\frac{-B k^{2}}{1+C_{\alpha} k^{2-\alpha}}\right) .
$$

In [20] it is shown that this expression is indeed the characteristic function of a probability density with power law tails whose exponent is exponent $5-\alpha$. However, this choice is problematic in two respects: its inverse Fourier cannot be computed explicitly, which prevents a fully explicit theory. In addition, for Eq. (1) to be consistent, $\tilde{g}\left(\sqrt{k_{1}^{2}+\cdots+k_{n}^{2}}\right)$ must be the characteristic function of a multivariate probability density for all $n$. In [16] only numerical checks are performed to verify this property. But as discussed for example in [2] both truncated Lévy and Student distributions yield acceptable fits of the returns on medium and small time scales. In the present context, the Student distribution, sometimes referred to as $q$-Gaussian in the case of non-integer degrees of freedom, is a better choice; it provides analytic tractability while fitting equally well real stock market prices (see also [21]). The fit of the daily returns of the $\mathrm{S} \& \mathrm{P} 500$ index in the period with a Student distribution

$$
g_{1}(x)=\frac{\Gamma\left(\frac{\nu}{2}+\frac{1}{2}\right)}{\pi^{1 / 2} \lambda \Gamma\left(\frac{\nu}{2}\right)} \frac{1}{\left(1+\frac{x^{2}}{\lambda^{2}}\right)^{\frac{\nu}{2}+\frac{1}{2}}}
$$




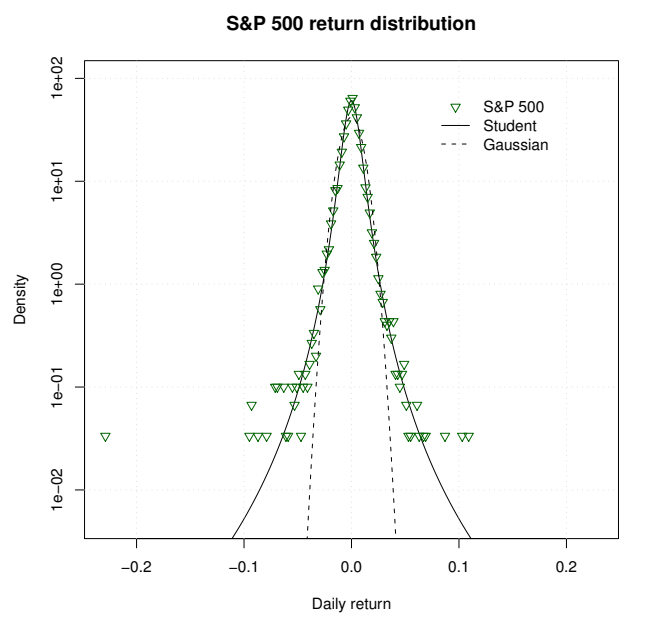

Fig. 1: Centered distribution of the 14956 daily returns of the S\&P 500 index (January, 3th 1950 - June, 11th 2009), and the corresponding fitting with Student $(\nu=3.21, \lambda=$ $0.0109)$ and Gaussian distribution $(\sigma=0.0095)$.

is reported in Fig. $1^{2}$.

The characteristic function of the Student density is

$$
\tilde{g}(k)=\frac{2^{1-\frac{\nu}{2}}}{\Gamma\left(\frac{\nu}{2}\right)} k^{\frac{\nu}{2}} K_{\frac{\nu}{2}}(k),
$$

where $K_{\alpha}$ is the modified Bessel function of third kind. As demonstrated in the appendix, the inverse Fourier transform of $\tilde{g}\left(\sqrt{k_{1}^{2}+\cdots+k_{n}^{2}}\right)$ for any integer $n$ is simply the multivariate Student distribution (see also [23]). The general form of this distribution can be written as

$$
g_{n}^{(\nu)}(\mathbf{x}, \boldsymbol{\Lambda})=\frac{\Gamma\left(\frac{\nu}{2}+\frac{n}{2}\right)}{\pi^{n / 2}(\operatorname{det} \boldsymbol{\Lambda})^{1 / 2} \Gamma\left(\frac{\nu}{2}\right)} \frac{1}{\left(1+\mathbf{x}^{t} \boldsymbol{\Lambda}^{-1} \mathbf{x}\right)^{\frac{\nu}{2}+\frac{n}{2}}},
$$

where $\nu>1$ is the exponent of the power law of the tails, $\mathcal{P}(r>R) \propto 1 / R^{\nu}$ and $\boldsymbol{\Lambda}$ is a positive definite symmetric matrix governing the variance-covariance matrix $E\left(x_{i}, x_{j}\right)=\frac{\Lambda_{i j}}{\nu-2}$, which does exist provided that $\nu>2$.

\footnotetext{
${ }^{2}$ All the graphics and numerical calculations have been per-
} formed with [22].
In passing, the same properties are shared by multivariate symmetric generalized hyperbolic distributions introduced in finance by [24] (see also [25]). The general case is obtained by an affine change of variable, but for the sake of brevity let us restrict to

$$
f(\mathbf{x})=\frac{\alpha^{\frac{n}{2}}}{(2 \pi)^{\frac{n}{2}} K_{\frac{\nu}{2}}(\alpha)} \frac{1}{\left(1+r^{2}\right)^{\frac{\nu}{4}+\frac{n}{4}}} K_{\frac{\nu}{2}+\frac{n}{2}}\left(\alpha \sqrt{1+r^{2}}\right)
$$

for $\mathbf{x} \in \mathbb{R}^{n}$ and $r$ the usual euclidean norm of $\mathbf{x}$. Student distributions are recovered in the limit $\alpha \rightarrow 0^{+}$. As shown in the appendix, its characteristic function is given for any $n$ by

$$
\tilde{f}_{n}(\mathbf{k})=\frac{K_{\frac{\nu}{2}}\left(\sqrt{\alpha^{2}+k^{2}}\right)}{K_{\frac{\nu}{2}}(\alpha)} \frac{\left(\alpha^{2}+k^{2}\right)^{\frac{\nu}{4}}}{\alpha^{\frac{\nu}{2}}}
$$

with $k=\sqrt{\sum_{i=1}^{n} k_{i}^{2}}$.

In the following we restrict the discussion to the Student distributions. Hence we assume that the distribution of the return is given by Eq. (7) with characteristic function given by Eq. (6), where $\boldsymbol{\Lambda}$ is a diagonal matrix

$$
\begin{aligned}
k & =\sqrt{\mathbf{k}^{t} \boldsymbol{\Lambda} \mathbf{k}} \\
& =\lambda \sqrt{k_{0}^{2}+\left(2^{2 D}-1\right) k_{1}^{2}+\cdots+\left(n^{2 D}-(n-1)^{2 D}\right) k_{n-1}^{2}}
\end{aligned}
$$

and $\lambda^{2}$ governs the variance of the returns on the time scale chosen as a reference. Thanks to the fact that the diagonal elements of $\boldsymbol{\Lambda}$ form a telescoping series the process is indeed consistent for any number of discrete steps. Moreover it can be generalized to the continuous time by setting, in the same consistent way,

$$
\begin{aligned}
& \mathcal{P}\left(r_{0, \Delta t_{0}}, r_{t_{1}, \Delta t_{1}}, \ldots, r_{t_{n-1}, \Delta t_{n-1}}\right) \\
& =g_{n}^{(\nu)}\left(r_{0, \Delta t_{0}}, r_{t_{1}, \Delta t_{1}}, \ldots, r_{t_{n-1}, \Delta t_{n-1}}, \boldsymbol{\Lambda}\right) \\
& \left.=\operatorname{diag}\left(t_{1}^{2 D}, t_{2}^{2 D}-t_{1}^{2 D}, \ldots, t_{n}^{2 D}-t_{n-1}^{2 D}\right)\right)
\end{aligned}
$$


where $t_{j}=\sum_{i=0}^{j-1} \Delta t_{i}, j \geq 1$ and now $\boldsymbol{\Lambda}=\operatorname{diag}\left(t_{1}^{2 D}, t_{2}^{2 D}-\right.$ $\left.t_{1}^{2 D}, \ldots, t_{n}^{2 D}-t_{n-1}^{2 D}\right)$. The existence of the continuum process is then guaranteed by the Kolmogorov extension theorem. Starting from this expression a wider class of processes can be generated by suitable transformations of the time, i.e., by substituting the function $t_{i} \rightarrow t_{i}^{2 D}$ for any monotonically increasing continuous function $t_{i} \rightarrow T\left(t_{i}\right)$. The process followed by the price $x(t)=\ln S(t)$ is a Student process too, with same exponent $\nu$ and non diagonal $\operatorname{matrix} \Lambda_{i j}=(-1)^{i+j} T\left(t_{\min (i, j)}\right)$.

The Student setting makes easier to interpret the correlations induced by the pointwise non-standard product of (2) in the characteristic function space. If we consider two variables $x_{1}$ and $x_{2}$ distributed according to $g_{1}(x)$, the joint probability function will be $g_{2}\left(x_{1}, x_{2}\right)$. The variables $X_{i}=G\left(x_{i}\right)=\int_{-\infty}^{x_{i}} d x g_{1}(x)$ are distributed uniformly on the interval $[0,1]$; by definition, the copula function $c\left(X_{1}, X_{2}\right)$ (cf. e.g. [26] for a general theory) is

$$
\begin{aligned}
c\left(X_{1}, X_{2}\right) & =g_{2}\left(G^{-1}\left(X_{1}\right), G^{-1}\left(X_{2}\right)\right) \frac{d x_{1}}{d X_{1}} \frac{d x_{2}}{d X_{2}} \\
& =\frac{g_{2}\left(G^{-1}\left(X_{1}\right), G^{-1}\left(X_{2}\right)\right)}{g\left(G^{-1}\left(X_{1}\right)\right) g\left(G^{-1}\left(X_{2}\right)\right)} .
\end{aligned}
$$

In our case $c$ is none other than the Student copula function, generally applied in finance for describing the correlation among asset prices $([27,28])$. A picture of this copula density with $\nu=3$ and $\boldsymbol{\Lambda}$ the identity matrix is given in Fig. 2. Although Student and generalized hyperbolic distributions are usually adopted for modeling returns of several assets over the same time intervals, the framework proposed by Baldovin and Stella allow them to model the returns of a single asset over different time intervals.

\section{The Baldovin-Stella Process as}

\section{Multivariate Normal Variance Mixtures}

According to the Ba-St framework we have to look for functions $\phi: \mathbb{R} \rightarrow \mathbb{C}$, such that $\tilde{g}_{n}: \mathbb{R}^{n} \rightarrow \mathbb{C}$ with $\tilde{g}_{n}\left(k_{1}, k_{2}, \ldots, k_{n}\right)=\phi\left(k_{1}^{2}+k_{2}^{2}+\cdots+k_{n}^{2}\right)$ is the characteristic function of a probability distribution for any $n$. Then from Eq. (8) we obtain a unique stochastic process with a well-defined continuous limit.

Ba-St processes can be fully characterized if one regards their finite dimensional marginals as instances of multivariate normal variance mixtures $U=\sigma N$, where $\sigma$ is an univariate random variable with positive values, $\sigma^{2}$ having cumulative distribution $G$, and $N$ is an $n$-dimensional normal random variable independent from $\sigma$. Leaving aside trivial affine changes of variables, we can assume that the covariance matrix of $N$ is the identity matrix. By first conditioning its evaluation on the value of $\sigma$, and then computing its mean over $\sigma$, it is immediate to see that the characteristic function $\tilde{g}_{n}^{U}\left(k_{1}, k_{2}, \ldots, k_{n}\right)$ of $U$ is

$$
\tilde{g}_{n}^{U}\left(k_{1}, k_{2}, \ldots, k_{n}\right)=\phi_{\sigma^{2}}\left(\frac{1}{2}\left(k_{1}^{2}+k_{2}^{2}+\cdots+k_{n}^{2}\right)\right)
$$

where $\phi_{\sigma^{2}}(s)$ is the Laplace transform associated to $G$

$$
\phi_{\sigma^{2}}(s)=\int_{0}^{\infty} d x e^{-s x} d G(x)
$$

As this construction is independent from $n$, an admissible choice for $\phi$ is $\phi(s)=\phi_{\sigma^{2}}\left(\frac{s}{2}\right)$, where $\phi_{\sigma^{2}}$ is the Laplace transform associated to any random variable $\sigma^{2}$ with positive values.

The crucial point is that by Schoenberg's theorem in [29] (see also the self-contained discussion about normal 


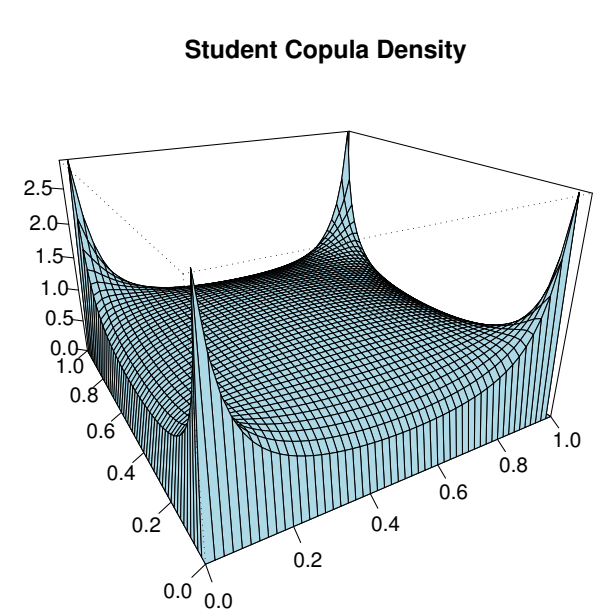

(a) $3 \mathrm{D}$ perspective.

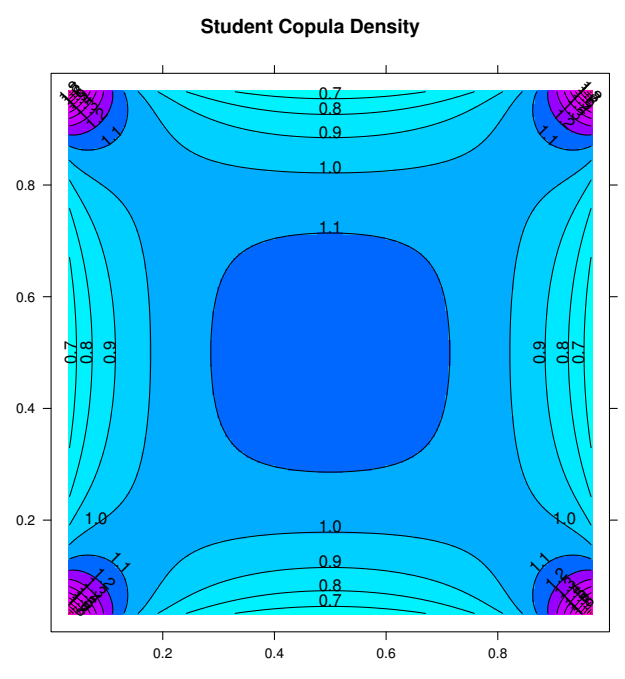

(b) Level plot.

Fig. 2: Student copula density with $\nu=3$ and trivial correlation matrix.

variance mixtures in [30]) this family exhausts all the possible choices, i.e. $\phi\left(k_{1}^{2}+k_{2}^{2}+\cdots+k_{n}^{2}\right)$ is a characteristic function of a probability distribution for any $n$ if and only if $\phi(s)$ is the Laplace transform a univariate random variable with positive values.

Hence a multivariate distribution for the returns can be built in the Ba-St framework if and only if it admits a representation as a normal variance mixture.

In passing we note that the choice of Ba-St in their original papers for the distribution (5) is indeed admissible, as in [20] it is shown that

$$
\phi_{S}(s)=\exp \left(\frac{-B s}{1+C_{\alpha} s^{1-\alpha / 2}}\right)
$$

is completely monotone, hence a Laplace transform by the virtue of Bernstein's theorem.
Now it is immediate to see that all the stochastic processes $X_{t}^{\sigma}(\omega)$ that can arise in the Ba-St framework admit the following representation on a suitably chosen filtered probability space $(\Omega, \mathcal{F}, \mathbb{P})$, over which a positive random variable $\sigma(\omega)$ and a Wiener process $W_{t}(\omega)$ independent from $\sigma$ are defined:

$$
X_{t}^{\sigma}(\omega)=\sigma(\omega) W_{t^{2 D}}(\omega)
$$

We only have to show that the finite dimensional marginal laws of $X_{t}^{\sigma}(\omega)$ are the same as those arising from (8). Indeed if we first evaluate the expectations over $W$, conditional on $\sigma$, we will obtain a Gaussian multivariate distri- 
bution

$$
\begin{aligned}
& \mathcal{P}\left(X_{t_{1}}, X_{t_{2}}, \ldots, X_{t_{n}} \mid \sigma\right) \\
&=\frac{1}{\left(2 \pi \sigma^{2}\right)^{\frac{n}{2}}} \exp \left[-\frac{1}{2 \sigma^{2}}\left(\frac{X_{t_{1}}^{2}}{t_{1}^{2 D}}+\frac{\left(X_{t_{2}}-X_{t_{1}}\right)^{2}}{t_{2}^{2 D}-t_{1}^{2 D}}\right.\right. \\
&\left.\left.\quad+\cdots+\frac{\left(X_{t_{n}}-X_{t_{n-1}}\right)^{2}}{t_{n}^{2 D}-t_{n-1}^{2 D}}\right)\right] ;
\end{aligned}
$$

the eventual average over $\sigma$ will then lead to the same multivariate normal variance mixtures as in (8), with the appropriate covariance matrix (just note that $\Delta t_{i}=t_{i+1}-t_{i}$, and $\left.r_{i, \Delta t_{i}}=X_{t_{i+1}}-X_{t_{i}}\right)$. In particular, the processes introduced in Sec. 2 correspond to an inverse Gamma distribution of $\sigma^{2}$ in the Student case, and a Generalized Inverse Gaussian distribution in the hyperbolic case.

The stochastic differential equation obeyed by (8) is

$$
d X_{t}^{\sigma}(\omega)=\sigma(\omega) t^{D-\frac{1}{2}} d W_{t}
$$

This equation shows that the volatility of the processes admissible in the Ba-St framework has a deterministic time dynamic, and that its source of randomness is just ascribable to its initial value.

Eventually we can conclude that a stochastic process is compatible with the Ba-St framework if and only if it is a variance mixture of Wiener processes whose variance is distributed according an arbitrary positive law, with a deterministic power law time change. This explains why using use this framework to model real price returns, one inevitably has to assume that the real price dynamics is composed by sequences of different realizations, as done by Ba-St. This is necessary not only because otherwise the model would predict a persistent and deterministic volatility decay for $D<1 / 2$, but also because $\sigma$ is fixed in each realization. The limitations of this kind of models in describing real returns will be made more manifest in the following section, but now we already know their mathematical foundations.

The asset prices can be modeled in an obvious arbitrage free way

$$
S(t, \omega)=S_{0} \exp \left(r t+\sigma(\omega) W_{t^{2 D}}(\omega)-\frac{1}{2} \sigma^{2}(\omega) t^{2 D}\right)
$$

with $r$ the fixed default free interest rate, and where we left the dependence on $\omega$ explicit in order to emphasise the fact that $\sigma$ is a random variable. The pricing of options is then the same as in the Black-Scholes model, with an additional average over $\sigma(\omega)$. For instance the price $C(T, K)$ of a call option with maturity $T$ and strike $K$ is

$$
C(T, K)=S_{0} E_{\sigma}\left(N\left(d_{1}\right)\right)-e^{-r T} K E_{\sigma}\left(N\left(d_{2}\right)\right),
$$

with as usual $N$ is the normal cumulative distribution,

$$
\begin{aligned}
& d_{1}=\frac{\ln \frac{S_{0}}{K}+r t+\frac{1}{2} \sigma^{2} t^{2 D}}{\sigma t^{D}}, \\
& d_{2}=\frac{\ln \frac{S_{0}}{K}+r t-\frac{1}{2} \sigma^{2} t^{2 D}}{\sigma t^{D}},
\end{aligned}
$$

and the additional expectation $E_{\sigma}$ has to be evaluated according to the distribution of $\sigma$.

\section{Applicability of this Framework to Real}

\section{Markets}

The axiomatic nature of the derivation of Baldovin and Stella is elegant and powerful: its ability to build mathematically multivariate price return distributions from a univariate distribution using only a few reasonable assumptions is impressive. Nevertheless, as stated in the introduction, a model of price dynamics must meet many 
requirements in order to be both relevant and useful. In this section, we examine its dynamics thoroughly.

\subsection{Volatility dynamics}

In Fig. 3.a we report the results of three simulations of the return process, each one of 500 steps and with parameters $\nu=3.2$ and $D=0.20$. In each run the volatility decays ineluctably, as explained in the previous section. Indeed by fixing the time interval $\delta t_{i}=1$, we see from Eq. (8) that the unconditional volatility of the $r_{t, 1}$ returns is proportional to $\sqrt{(t+1)^{2 D}-t^{2 D}}$, i.e., to $t^{D-1 / 2}$ for $t \gg 1$ : the unconditional volatility decreases if $D<1 / 2$ and increases if $D>1 / 2$, in both cases according to a power law. This appears quite clearly in Fig. 3.b, where we have computed the mean volatility decay, measured as the absolute values of the return, over 10000 process simulations. The parameters of the distributions have been chosen close to those representing real returns (see below).

The conditional volatility can be easily computed: the distribution of the return $r_{n, 1}$ conditioned to the previous return realizations $r_{0,1}, \ldots, r_{n-1,1}$ is again a Student distribution with exponent $\nu \prime=\nu+n$ and conditional variance

$$
\left[(n+1)^{2 D}-n^{2 D}\right]\left(1+\sum_{i=0}^{n-1} \frac{r_{i, 1}^{2}}{(i+1)^{2 D}-i^{2 D}}\right)
$$

From this expression it is clear that volatility spikes in a given realisation of the process tend to be persistent (see Fig. 3.a); this is the main reason why fluctuation patterns differ much from one run to an other. This can be also understood by appealing to the characterization of this kind of processes we did in Sec. 3: each single run is just a realization of a Wiener process, whose variance is chosen at the beginning according to an Inverse Gamma distribution $R \Gamma\left(\frac{\nu}{2}, \frac{\lambda}{2}\right)$, and that decays in time according to the deterministic law $t^{D-\frac{1}{2}}$.

\subsection{Decreasing volatility and restarts}

The very first model introduced by Ba-St has constant volatility, which corresponds to $\boldsymbol{\Lambda}$ being a multiple of the identity matrix. This unfortunate feature is the main reason behind the introduction of weights, whose effect is akin to an algebraic stretching of the time, or, as put forward by Ba-St, to a time renormalization. This in turn causes a deterministic algebraic decrease of the expectation of the volatility, as explained above and depicted in Fig. 3.b; hence the need for restarts, each attributed to an external cause.

Although this dynamics may seem quite peculiar, such restarts are found at market crashes, like the recent one of October 2008, which are followed by periods of algebraically decaying volatility. This leads to an analogous of the Omori law for earthquakes, as reported in [31] and [32]. The Ba-St model, by construction, is able to reproduce this effect in a faithfully way. In Fig. 4 the cumulative number of times the absolute value of the returns $N(t)$ exceeds a given thresholds is depicted, for a single simulation of the process and three different value of the threshold. The fit with the prediction of the Omori law $N(t)=K\left(t+t_{0}\right)^{\alpha}-K t_{0}^{\alpha}$ is evident. 


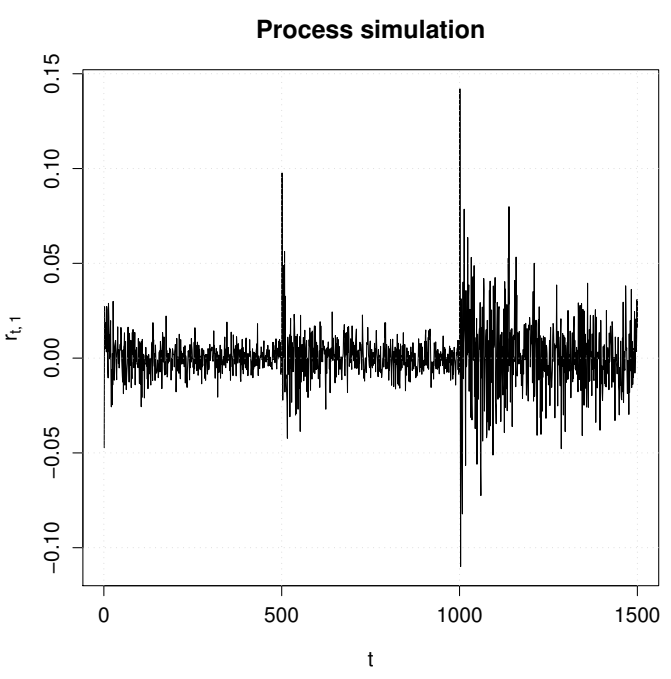

(a) Three simulations, each 500 steps long.

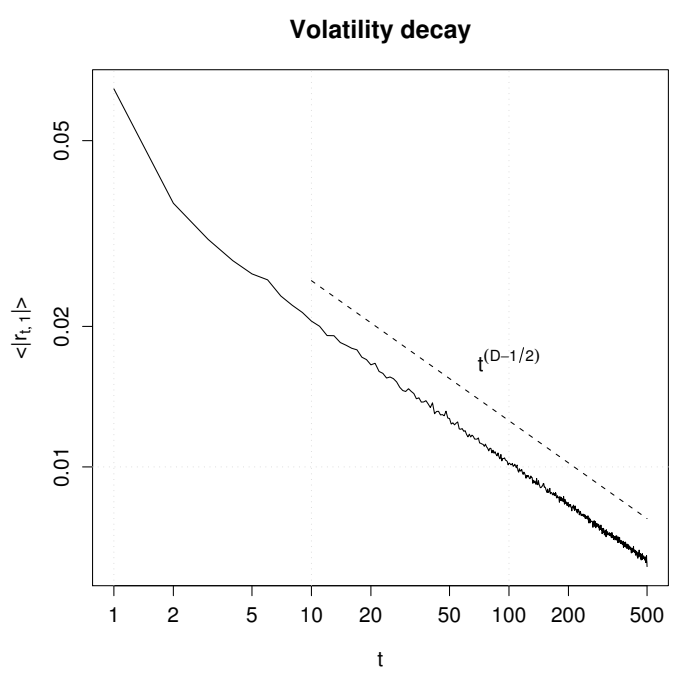

(b) Decay of the volatility: average over 10000 simulation, each 500 steps long. The dashed line represents the analytic prediction.

Fig. 3: Process simulation with $\nu=3.2, D=0.20$, and $\lambda=0.107$.

Crashes are good restart candidates: they provide clearly $D=1-2 \eta=0.3$. No explanation of why the trading acdefined events that synchronize all the traders' actions. In that view, they provide an other indirect way to measure the distribution of timescales of traders, which are thought to be power-law distributed $([33])$.

Another example of algebraically decreasing volatility was recently reported by [34] in foreign exchange markets in which trading is performed around the clock. Understandably, when a given market zone (Asia, Europe, America) opens, an increase of activity is seen, and viceversa. Specifically, this work fits the decrease of activity corresponding to the afternoon trading session in the USA with a power-law and finds an algebraic decay with exponent $\eta=0.35$; this is exactly the same behavior as the one of Ba-St model between two restarts, with tivity should result in this specific type of decay has been put forward in our knowledge. In this case the starting time of the volatility decay corresponds to the maximum of activity of US markets.

At any rate, restarts are a simple way to keep the dynamics alive. In real markets however, there is no reason to assume that $D$ is time independent. For instance, Ref. [35] measures a quantity related to $D$ as a function of time and finds a non-trivial time dependence, particularly in times of crisis. 

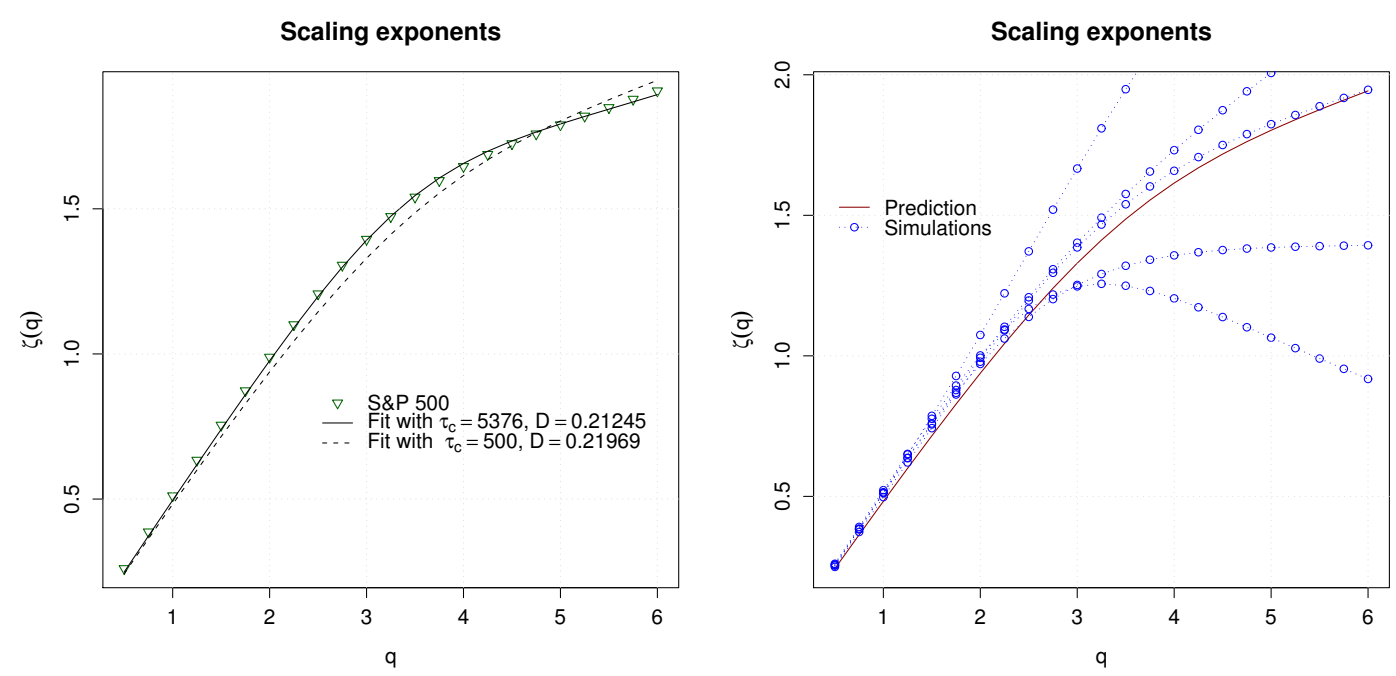

(a) Fitting of the empirical exponents of real data.(b) Theoretical prediction compared to 5 simulations done with the same parameters.

Fig. 5: Scaling exponents: S\&P 500 data and simulations compared with theoretical prediction. All the simulations have been done with the same parameters: 30 runs of 500 steps, with $\nu=3.2, D=0.220$

\subsection{Apparent multifractality}

The Baldovin and Stella model is able to reproduce the apparent multifractal characteristics of the real returns, i.e. the shape of $\zeta(q)$ where $\left\langle\left|r_{\delta t}\right|^{q}\right\rangle=\delta t^{\zeta(q)}$.

The expectation is evaluated according the distribution (4), i.e. taking the mean over independent runs of the process. Hence the expectation of the $q$ th moment in this model is

$$
\left\langle|r|^{q}\right\rangle_{\bar{P}_{\delta t}}=\frac{\left\langle|r|^{q}\right\rangle_{P_{t=0, \delta t=1}}}{\tau_{c}} \sum_{t=0}^{\tau_{c}-1}\left[(t+\delta t)^{2 D}-t^{2 D}\right]^{q / 2}
$$

(see the addendum to [16]). The exponents $\zeta(q)$ are evaluated as the slopes of the linear fitting of $\ln \left(\left\langle|r|^{q}\right\rangle_{\bar{P}_{\delta t}}\right)$ with respect to $\ln (\delta t)$. Hence in our case they are determined by the expression $\ln \sum_{t=0}^{\tau_{c}-1}\left[(t+\delta t)^{2 D}-t^{2 D}\right]^{q / 2}$, and depend only on $D$ and $\tau_{c}$. In Fig. 5.a is depicted the fitting of the S\&P 500 exponents with the model (9). The best fit is obtained with $D=0.212$ and $\tau_{c}=5376$. Unfortunately a value of $\tau_{c}$ that large is difficult to justify, as in the case of S\&P 500 we have only 14956 daily returns, i.e. less than three runs of a process with such a length. The other fit is obtained by first fixing $\tau_{c}=500$, as in [16] and yields $D=0.220$.

The statistical significance of this approach seems anyway questionable. In Fig. 5.b we compare the theoretical expectation of the exponents with simulations. We choose the parameters $\tau_{c}=500, D=0.220$ both for simulations and analytic model, with $\nu=3.22$. The number of restarts in the simulation is 30 in order to have a number of data points similar to the S\&P 500. It is evident that the ex- 


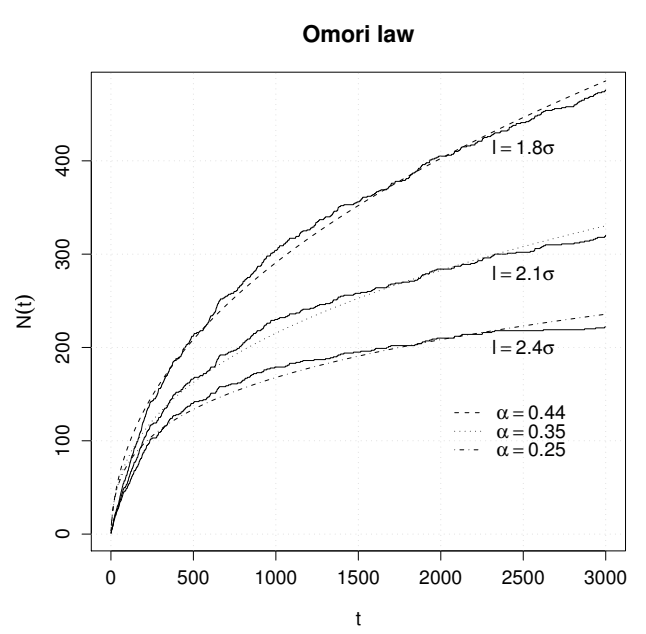

Fig. 4: Omori law for a single run of the process, with $D=0.20, \nu=0.32 . N(t)$ is the cumulative number the absolute value of the return exceeds a given thresholds. Three different values of the threshold $l$ have been chosen, measured with respect to the standard deviation $\sigma$ of the data. The dashed lines represents the fit with the Omori law $N(t)=K\left(t+t_{0}\right)^{\alpha}-K t_{0}^{\alpha}$.

ponents evaluated from the simulated data have a really large variance.

The problem is that if the tail exponent $\nu=3.22$, from an analytic perspective the moments with $q>3.22$ are infinite, hence, should not be taken into account in the multifractal analysis (for an analytic treatment of multifractal analysis see [36-38]). The situation is somehow different in the case of multifractal models of asset returns ([39,6]), where the theoretical prediction of the tail exponents of the return distribution is relatively high (see the review of [9]), and the moments usually empirically measured do exist even from the analytic point of view. For attempts to reconcile the theoretical predictions of the multifractal models with real data see [40] and [41].

It is worth remembering that the anomalous scaling of the empirical return moments does not imply that the return series has to be described by a multifractal model, as already pointed out some time ago in [42] and [43]: the long memory of the volatility is responsible at least in part for the deviation from trivial scaling. A more detailed analysis of real data reported in [44] seems indeed to exclude evident multifractal properties of the price series.

\section{Missing Features}

Since in this model the volatility is constant in each realization and bound to decrease unless a restart occurs, it is quite clear that it does not contain all the richness of financial market price dynamics. Restarting the whole process is not entirely satisfactory, as in reality the increase of volatility is not always due to an external shock. Volatility does often gradually build up through a feedback loop that is absent from the Ba-St mechanism. Thus, large events and crashes can also have a endogenous cause, e.g. due to the influence of traders that base their decisions on previous prices or volatility, such as technical analysts or hedgers. A quantitative description of this kind of phenomena is attempted for instance in $[45,46]$, by appealing to discrete scale invariance (see also the viewpoint expressed in [47] and references therein). This kind of effect is completely missing from the original Ba-St mechanism.

Volatility build-ups can be simulated with $D>1 / 2$, getting at constant $D$ the equivalent of the inverse Omori 
law for earthquakes [48]. This kind of dynamics has been reported to happen prior to some financial market crashes [46]. At a smaller time scale, foreign exchange intraday volatility patterns have a systematically increasing part whose fit to a possibly arbitrary power-law, as performed in $[34](\eta=0.22)$, corresponds indeed to choosing $D=$ 0.56. To our knowledge, volatility build-ups either do not follow a particular and systematic law, or perhaps have not yet been the objects of a thorough study.

Because of the symmetric nature of all the distributions derived above, all the odd moments are zero, hence, the skewness of real prices cannot be reproduced. This shows up well in Fig. 3 of [17]. Another consequence is that it is impossible to replicate the leverage effect, i.e. the negative correlation between past returns and future volatility, carefully analyzed in [49].

In any case, the decrease of the fluctuations in the BaSt process is a deterministic outcome of the anomalous scaling law $t^{D}$ with $D<1 / 2$, and results in a strong temporal asymmetry of the corresponding time series. But quite remarkably it misses the time-reversal asymmetry reported in [50] and [51]. Indeed real financial time series are not symmetric under time reversal with respect to even-order moments. For instance, there is no leverage effect in foreign exchange rates, and their time series are not as skewed as indices, but they do have a time arrow. One of the indicators proposed in [50] is the correlation between historical volatility $\sigma_{\delta t_{h}}^{(h)}(t)$ and realized volatility $\sigma_{\delta t_{r}}^{(r)}(t)$. The historical volatility series $\sigma_{\delta t_{h}}^{(h)}(t)$ represents the volatility computed using the data in the past interval $\left[t-\delta t_{h}, t\right]$, and $\sigma_{\delta t_{r}}^{(r)}(t)$ represents the volatility computed using the data in the future interval $\left[t, t+\delta t_{r}\right]$; the correlation between the two series is then analyzed as a function of both $\delta t_{r}$ and $\delta t_{h}$. Real financial time series present an asymmetric graph with respect the change $\delta t_{h} \leftrightarrow \delta t_{s}$, with a strong indication that historical volatility at a given time scale $\delta t_{h}$ is more likely correlated to realized volatility with time scale $\delta t_{r}<\delta t_{h}$, with peaks of correlation at time scales related to human activities. The asymmetry characteristic is absent in the Baldovin and Stella model, as showed in Fig. 6.

Without random restarts, the strong correlation between returns guarantees the slow decay of the volatility but induces some side effects. The distribution of the returns in the model is essentially the same with identical power law exponent for the tails. This happens independently of the time interval $\delta t$ over which the returns are evaluated, as long as $\delta t \ll \tau_{c}$, with $\tau_{c}$ of the order of hundreds days. Hence the weekly returns are distributed as the daily returns, while in real data the tail exponent begins to increase in a remarkable way already at the intraday level ([52]). The strong correlation also slows down the convergence to the Gaussian distribution of the returns when measured on larger time scale. Even if the kurtosis is not defined analytically in principle, it is possible to measure the empirical kurtosis of the returns of a simulated time series and compare with the kurtosis of real data. In Fig. 7 we show the kurtosis of the return distribution among simulations and daily return of the S\&P 500 index; the kurtosis has been computed for the returns 


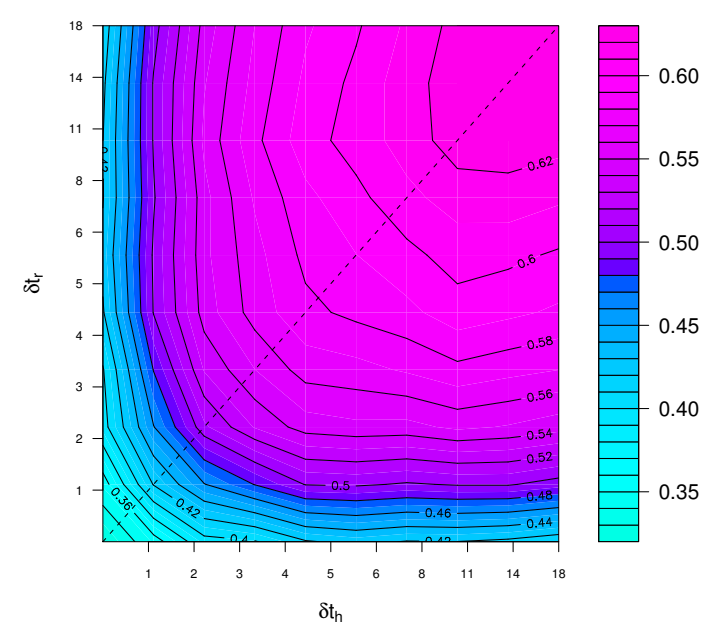

(a)

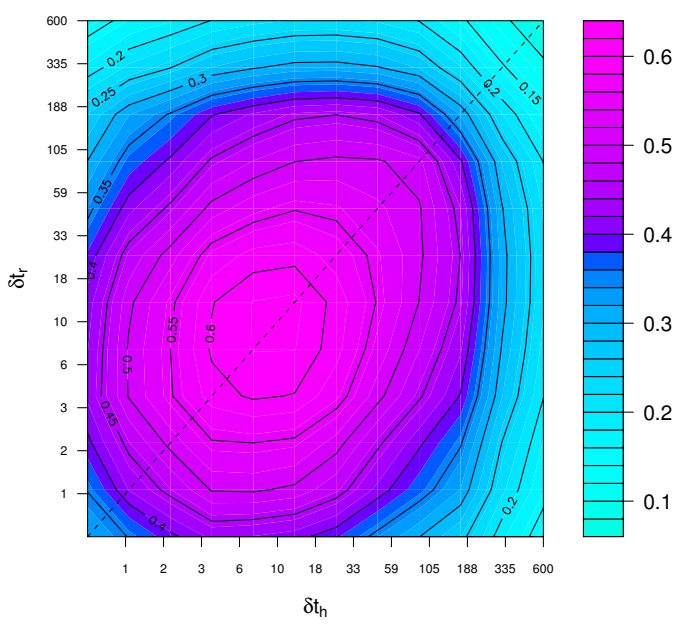

(b)

Fig. 6: Correlation between historical and realized volatility of the simulated process, over different time interval $\delta t$. The analyzed time series was composed by 1000 runs of the basic process, each one with 200 steps, and parameter $\nu=3.22, D=0.20$.

over different interval $\delta t$, and the simulated processes had the same length (30 runs of 500 steps) of the real series.

\section{Possible Improvements}

The main limitations of the model originally proposed by Baldovin and Stella are poor volatility dynamics, lack of skewness, some unwanted symmetry with respect to time, and slow convergence to a Gaussian. In addition to random restarts, we think it worthwhile considering other modifications.

The volatility dynamics can be improved by introducing an appropriate dynamics for the exponent $D$, i.e. introducing a dynamic $D(t)$ controlling the diffusive process. This is equivalent to starting with a model with constant volatility, i.e. with $\boldsymbol{\Lambda}$ proportional to the identity matrix,

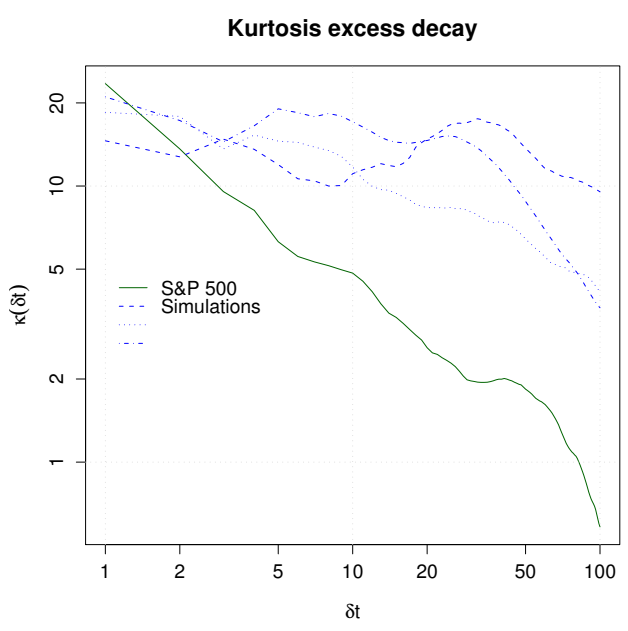

Fig. 7: Comparison of the kurtosis of the returns evaluated over a time interval $\delta t$. Each one of the three simulations are composed by 30 runs, 500 steps long, in order to have a length comparable with that of the S\&P 500 returns. The parameters are $\nu=3.2, D=0.20, \lambda=0.1$. 
and then introducing an appropriate evolution for the time $t$. This technique is employed for instance in the Multifractal Random Walk model ([39]), where the time evolution is driven by a multifractal process, or when the time evolution is modeled by an increasing Lévy process (see e.g. [13]). In this last case we would obtain a mixing of Wiener processes driven by a subordinator.

The lack of skewness is a common problem of stochastic volatility models: one usually writes the return at time $t$ as $r_{t, \delta t}=\epsilon(t) \sigma(t)$, where $\epsilon(t)$ is sign of the return and $\sigma(t)$ its amplitude, a symmetric setting if the distribution of $\epsilon(t)$ is even. One remedy found for instance in [8] is to bias the sign probabilities while enforcing a zero expectation; more precisely,

$$
P\left(\epsilon= \pm \frac{1 / \sqrt{2}}{1 / 2 \pm \epsilon}\right)=1 / 2 \pm \epsilon .
$$

Another possibility for introducing skewness is that of considering normal mean-variance mixtures, instead of simply normal variance ones. For instance, this would have implied the use of the multivariate skewed Student distribution in the model described in Sec. 2 .

The decay of the tail exponent of the return distribution, represented in Fig. 7, could be implemented by introducing two different Student distributions: a univariate with exponent $\nu_{r}$ for modeling the daily returns, and a multivariate one with a much larger exponent $\nu_{c}$ for modeling the correlations among them. By taking into account the generalized central limit theorem expressed in Eq. (3), the distribution of returns at intermediate time scales will interpolate between the two exponents, yielding the desired feature.
The Zumbach mugshot is one of the most difficult stylized facts to reproduce. To our knowledge the best results in that respect was achieved in [12], where a specific realization of a quadratic GARCH model is introduced, motivated by the different activity levels of traders with different investment time horizons, which take into account the return over a large spectrum of time scales. More specifically Borland and Bouchaud use

$$
\sigma_{i}^{2}=\sigma_{0}^{2}\left[1+\sum_{\delta t=1}^{\infty} g_{\Delta t} \frac{r_{i, \delta t}^{2}}{\sigma_{0}^{2} \tau \delta t}\right],
$$

with $\tau$ fixing the time scale, $r_{t, \delta T}=\ln S(t+\delta T)-\ln S(t)$, $g_{\delta t}$ measuring the impact on the volatility by traders with time horizon $\delta t$, and chosen by the authors $g_{\delta t}=g /(\delta t)^{\alpha}$. This expression is rewritten also in the form

$$
\sigma_{i}^{2}=\sigma_{0}^{2}+\sum_{j<i, k<i} \mathcal{M}(i, j, k) \frac{r_{j} r_{k}}{\tau}
$$

with

$$
\mathcal{M}(i, j, k)=\sum_{\Delta t=\max (i-j, i-k)}^{\infty} \frac{g_{\delta t}}{\delta t} .
$$

In the present framework this would correspond to use a highly non-trivial matrix $\boldsymbol{\Lambda}$, introducing linear correlation among returns at any time lag. This means that the Ba-St process would no longer be a model of returns, but of stochastic volatility.

\section{Discussion and Conclusions}

When employed with self-decomposable distributions like the Student or the Generalized Hyperbolic as introduced in Sec. 2, the resulting description of the process return is different than that of other models in the literature. First 
our Student process is not stationary, hence different from the class of Student processes discussed in [53], where the main focus is on stationary ones. The processes (8) are also different from the one studied in [54]: the latter too are continuous and based on the Student distributions, but defined by the stochastic differential equation

$$
d X_{t}=t^{D-\frac{1}{2}} \sqrt{\frac{2 D c_{0}}{\nu-1}} \sqrt{1+\frac{X_{t}^{2}}{c_{0} t^{2 D}}} d W
$$

apart from the striking difference with Eq. (8), in [55] it is shown that not all the marginal distribution laws of $X_{t}$ are of Student type.

Instead in [24] the Generalized Hyperbolic laws are adopted for describing the returns at a fixed time scale; these laws are then extended to the other time scales using the standard Lévy process construction: in this case the distributions at the other time scales are no more of Generalized Hyperbolic type.

The Baldovin and Stella model is also intrinsically simpler than the ones described in [56], where the volatility has a dynamic modeled by Ornstein-Uhlenbeck type processes,

$$
d \sigma_{t}^{2}=-\lambda \sigma_{t}^{2} d t+d L_{t}
$$

driven by an arbitrary Lévy process $L_{t}$. In this case, according to the choice of $L_{t}$, any self-decomposable distribution (like the Generalized Inverse Gaussian, or any of its special cases, like the Inverse Gamma) can arise as the distribution of $\sigma_{t}^{2}$ for any $t$. But this simplification comes at a high price: while in Barndorff-Nielsen $\sigma$ is truly dynamic, it is fixed in Ba-St for any single process realization.
In addition, the models analyzed in [14] are of a different type, even if there are some analogies in the underlying principles. In [14] indeed an anomalous scaling is introduced by considering self-similar processes, and in that framework any self-decomposable distribution can employed for modeling returns, but once again only at a fixed time scale, as in the standard case of Lévy processes. The main difference is that in [14] the returns at different times are assumed to be totally independent, but not identically distributed: instead Baldovin and Stella assume that the returns are only linearly independent, but now with identical distributions at all the time scales, up to a simple rescaling.

In conclusion, despite its current inability to reproduce all the needed stylized facts, the new framework proposed by Baldovin and Stella introduces a new mechanism for modeling returns, based on a few reasonable first principles. We therefore think that, once suitably modified for instance along the lines proposed above, the Ba-St framework can provide a new tool for building models of financial price dynamics from reasonable assumptions.

\section{Appendix: Some Useful Facts About Student} and Symmetric Generalized Hyperbolic Dis-

\section{tributions}

\section{Characteristic function of Student distributions}

The standard form of univariate Student distribution is

$$
g_{1}(x)=\frac{\Gamma\left(\frac{\nu}{2}+\frac{1}{2}\right)}{\pi^{1 / 2} \Gamma\left(\frac{\nu}{2}\right)} \frac{1}{\left(1+x^{2}\right)^{\frac{\nu}{2}+\frac{1}{2}}},
$$


while the multivariate one is

$$
g_{n}(\mathbf{x})=\frac{\Gamma\left(\frac{\nu}{2}+\frac{n}{2}\right)}{\pi^{n / 2} \Gamma\left(\frac{\nu}{2}\right)} \frac{1}{\left(1+r^{2}\right)^{\frac{\nu}{2}+\frac{n}{2}}}
$$

with $r=\sqrt{\sum_{i=1}^{n} x_{i}^{2}}$ and $\mathcal{P}(r>R) \propto 1 / R^{v}$.

Using some standard relationships involving Bessel functions one can compute analytically the corresponding characteristic function:

$$
\begin{array}{r}
\tilde{g}_{1}\left(k_{1}\right)=\int_{-\infty}^{+\infty} d x_{1} e^{i k_{1} x_{1}} g_{1}\left(x_{1}\right) \\
=\frac{2 \Gamma\left(\frac{\nu}{2}+\frac{1}{2}\right)}{\pi^{1 / 2} \Gamma\left(\frac{\nu}{2}\right)} k^{\nu} \int_{0}^{+\infty} d x\left(k^{2}+x^{2}\right)^{-\frac{\nu}{2}-\frac{1}{2}} \cos (x) \\
=\frac{2^{1-\frac{\nu}{2}}}{\Gamma\left(\frac{\nu}{2}\right)} k^{\frac{\nu}{2}} K_{\frac{\nu}{2}}(k),
\end{array}
$$

with $k=\left|k_{1}\right|, K_{\alpha}$ the modified Bessel function of third kind, and the employ of identity 7.12.(27) of [57]

$$
\begin{array}{r}
K_{\nu}(z)=\frac{(2 z)^{\nu}}{\pi^{1 / 2}} \Gamma\left(\nu+\frac{1}{2}\right) \int_{0}^{\infty} d t\left(t^{2}+z^{2}\right)^{-\nu-1 / 2} \cos (t) \\
\Re(\nu)>-\frac{1}{2},|\arg (z)|<\frac{\pi}{2} .
\end{array}
$$

For an alternative derivation we refer to [58] and to the discussion in [53]. An alternative expression is found in [59].

For general $n$ we obtain again the same expression. Indeed

$$
\begin{aligned}
\tilde{g}_{n}(\mathbf{k}) & =\int_{\mathbb{R}^{n}} d^{n} \mathbf{x} e^{i \mathbf{k} \cdot \mathbf{x}} g_{n}(\mathbf{x}) \\
& =\frac{\Gamma\left(\frac{\nu}{2}+\frac{n}{2}\right)}{\pi^{n / 2} \Gamma\left(\frac{\nu}{2}\right)} \int d^{n-2} \Omega \int_{0}^{+\infty} d r r^{n-1} \\
& \int_{0}^{\pi} d \phi \sin ^{n-2}(\phi) e^{i k r \cos \phi}\left(1+r^{2}\right)^{-\frac{\nu}{2}-\frac{n}{2}} \\
& =\frac{2^{n / 2} \Gamma\left(\frac{\nu+n}{2}\right)}{\Gamma\left(\frac{\nu}{2}\right)} k^{1-n / 2} \\
\int_{0}^{+\infty} d r r^{n / 2}\left(1+r^{2}\right)^{-\frac{\nu}{2}-\frac{n}{2}} J_{n / 2-1}(k r) & \frac{2^{1-\frac{\nu}{2}}}{\Gamma\left(\frac{\nu}{2}\right)} k^{\frac{\nu}{2}} K_{\frac{\nu}{2}}(k),
\end{aligned}
$$

with $k=\sqrt{\sum_{i=1}^{n} k_{i}^{2}}, d^{n-2} \Omega$ the surface element of the sphere $S^{n-2}, \phi$ the angle between $\mathbf{k}$ and $\mathbf{x}$ and the employ of identities 7.12.(9)

$$
\begin{array}{r}
\Gamma\left(\nu+\frac{1}{2}\right) J_{\nu}(z)=\frac{1}{\pi^{1 / 2}}\left(\frac{z}{2}\right)^{\nu} \int_{0}^{\pi} d \phi e^{i z \cos \phi}(\sin \phi)^{2 \nu} \\
\Re(\nu)>-\frac{1}{2}
\end{array}
$$

and 7.14.(51) of [57],

$$
\begin{gathered}
\int_{0}^{\infty} d t J_{\mu}(b t)\left(t^{2}+z^{2}\right)^{-\nu} t^{\mu+1}=\left(\frac{b}{2}\right)^{\nu-1} \frac{z^{1+\mu-\nu}}{\Gamma(\nu)} K_{\nu-\mu-1}(b z) \\
\Re\left(2 \nu-\frac{1}{2}\right)>\Re(\mu)>-1, \Re(z)>0 .
\end{gathered}
$$

Eventually one finds

$$
\tilde{g}_{n}(\mathbf{k})=\tilde{g}_{1}\left(\sqrt{k_{1}^{2}+\cdots+k_{2}^{2}}\right) .
$$

With the linear change of variables $\mathbf{x} \rightarrow \mathbf{C}^{-1} \mathbf{x}$, setting $\boldsymbol{\Lambda}^{-1}=\left(\mathbf{C}^{T}\right)^{-1} \mathbf{C}^{-1}$, i.e. $\boldsymbol{\Lambda}=\mathbf{C C}^{T}$, one obtains the following generalizations:

$$
g_{n}(\mathbf{x})=\frac{\Gamma\left(\frac{\nu}{2}+\frac{n}{2}\right)}{\pi^{n / 2}(\operatorname{det} \boldsymbol{\Lambda})^{1 / 2} \Gamma\left(\frac{\nu}{2}\right)} \frac{1}{\left(1+\mathbf{x}^{t} \boldsymbol{\Lambda}^{-1} \mathbf{x}\right)^{\frac{\nu}{2}+\frac{n}{2}}},
$$

with characteristic function

$$
\tilde{g_{n}}(\mathbf{k})=\frac{2^{1-\frac{\nu}{2}}}{\Gamma\left(\frac{\nu}{2}\right)}\left(\mathbf{k}^{t} \boldsymbol{\Lambda} \mathbf{k}\right)^{\frac{\nu}{4}} K_{\frac{\nu}{2}}\left(\left(\mathbf{k}^{t} \boldsymbol{\Lambda} \mathbf{k}\right)^{1 / 2}\right) .
$$

In the univariate case $\boldsymbol{\Lambda}$ is substituted by the scalar $\lambda^{2}$ and the previous expressions reduce to

$$
g_{1}(x)=\frac{\Gamma\left(\frac{\nu}{2}+\frac{1}{2}\right)}{\pi^{1 / 2} \lambda \Gamma\left(\frac{\nu}{2}\right)} \frac{1}{\left(1+\frac{x^{2}}{\lambda^{2}}\right)^{\frac{\nu}{2}+\frac{1}{2}}}
$$

and

$$
\tilde{g}_{1}(k)=\frac{2^{1-\frac{\nu}{2}}}{\Gamma\left(\frac{\nu}{2}\right)}(\lambda k)^{\frac{\nu}{2}} K_{\frac{\nu}{2}}(\lambda k)
$$

\section{Moments of Student distributions}

Due to the symmetry under reflection all the odd moments vanish. For the second moments we have, provided that 
$\nu>2$

$$
E\left(x_{i}, x_{j}\right)=\frac{\Lambda_{i j}}{\nu-2}
$$

The moments of order $2 n$ exist provided that $\nu>2 n$; as happens for Gaussian distributions, they can be expressed in term of the second moments,

$$
\begin{aligned}
& E\left(x_{j_{1}}, x_{j_{2}}, \ldots, x_{j_{2 n}}\right)= \\
& \quad \frac{\Gamma\left(\frac{\nu}{2}-n\right)}{2^{n} \Gamma\left(\frac{\nu}{2}\right)} \prod_{\text {all the pairings }} \Lambda_{j_{i_{1}} j_{i_{2}}} \cdots \Lambda_{j_{i_{2 n-1}} j_{i_{2 n}}} .
\end{aligned}
$$

In the univariate case these formulas reduce to $E\left(x^{2}\right)=$ $\frac{\lambda^{2}}{\nu-2}$ and

$$
E\left(x^{2 n}\right)=\frac{(2 n-1) ! ! \Gamma\left(\frac{\nu}{2}-n\right)}{2^{n} \Gamma\left(\frac{\nu}{2}\right)} \lambda^{2 n} .
$$

The kurtosis is then $\kappa=3 \frac{\nu-2}{\nu-4}$, provided that $\nu>4$.

\section{Simulation of multivariate Student distributions}

The simulation is a standard application of the technique used in the case of rotational invariance. From

$$
g_{n}(\mathbf{x}) d^{n} \mathbf{x}=\frac{\Gamma\left(\frac{\nu}{2}+\frac{n}{2}\right)}{\pi^{n / 2} \Gamma\left(\frac{\nu}{2}\right)} r^{n-1}\left(1+r^{2}\right)^{\frac{1}{1-q}} d^{n-1} \Omega d r
$$

with $r \geq 0$, we see that the density of the angular variables is uniform, while setting $y=\frac{r^{2}}{1+r^{2}}$, with $1>y \geq 0$ and $r=\sqrt{y /(1-y)}$, the density of $y$ is given by

$$
\frac{1}{B\left(\frac{n}{2}, \frac{\nu}{2}\right)} y^{\frac{n}{2}-1}(1-y)^{\frac{\nu}{2}-1} d y
$$

i.e. by the beta distribution with parameters $\frac{n}{2}$ and $\frac{\nu}{2}$. Eventually we can simulate the multivariate $n$ dimensional distribution by

1. Simulating $y$ according to $B_{x}\left(\frac{n}{2}, \frac{\nu}{2}\right)$ and setting $r=$ $\sqrt{\frac{y}{1-y}}$
2. Simulating $n$ i.i.d. Gaussian variables $u_{i}$ and settings

$$
\mathbf{n}=\left(u_{1}, \ldots, u_{n}\right) / \sqrt{u_{1}^{2}+\cdots+u_{n}^{2}} .
$$

3. Returning $x \mathbf{n}$.

The more general case (11) is simulated using the same algorithm and then returning $\mathbf{C x}$, where $\mathbf{\Lambda}^{-1}=\left(\mathbf{C}^{T}\right)^{-1} \mathbf{C}^{-1}$ i.e. $\boldsymbol{\Lambda}=\mathbf{C C}^{T}$.

\section{Characteristic function of symmetric generalized hyper-}

\section{bolic distributions}

We start from the expression

$$
f_{n}(\mathbf{x})=\frac{\alpha^{\frac{n}{2}}}{(2 \pi)^{\frac{n}{2}} K_{\frac{\nu}{2}}(\alpha)} \frac{K_{\frac{\nu}{2}+\frac{n}{2}}\left(\alpha \sqrt{1+r^{2}}\right)}{\left(1+r^{2}\right)^{\frac{\nu}{4}+\frac{n}{4}}}
$$

with $r=\sqrt{\sum_{i=1}^{n} x_{i}^{2}}$; the general case is obtained simply with an affine transformation $\mathbf{x} \rightarrow \mu+\delta \mathbf{R x}$, with $\mu \in \mathbb{R}^{n}$, $\delta \geq 0$ a scale parameter, and $\mathbf{R}$ an orthogonal transformation in $\mathbb{R}^{n}$. The central expression we need is an integral of the Sonine-Gegenbauer type, cf. identity 7.14.(46) of [57]:

$$
\begin{array}{r}
\int_{0}^{\infty} d t J_{\mu}(b t) K_{\nu}\left(a \sqrt{t^{2}+z^{2}}\right)\left(t^{2}+z^{2}\right)^{-\frac{\nu}{2}} t^{\mu+1} \\
=b^{\mu} a^{-\nu} z^{\mu-\nu+1}\left(a^{2}+b^{2}\right)^{\frac{\nu}{2}-\frac{\mu}{2}-\frac{1}{2}} K_{\nu-\mu-1}\left(z \sqrt{a^{2}+b^{2}}\right) \\
\Re(\mu)>-1, \Re(z)>0 .
\end{array}
$$

For $n=1$, considering that $J_{-\frac{1}{2}}(x)=\sqrt{\frac{2}{\pi x}} \cos (x)$, we obtain

$$
\tilde{f}_{1}\left(k_{1}\right)=\frac{K_{\frac{\nu}{2}}\left(\sqrt{\alpha^{2}+k_{1}^{2}}\right)}{K_{\frac{\nu}{2}}(\alpha)} \frac{\left(\alpha^{2}+k_{1}^{2}\right)^{\frac{\nu}{4}}}{\alpha^{\frac{\nu}{2}}} .
$$

For alternative derivations in the univariate case see [58] and the references therein.

In our setting the computation is exactly the same for general $n$, with $k=\sqrt{\sum_{i=1}^{n} k_{i}^{2}}, d^{n-2} \Omega$ the surface element 
of the sphere $S^{n-2}, \phi$ the angle between $\mathbf{k}$ and $\mathbf{x}$, using identity (10)

$$
\tilde{f}_{n}(\mathbf{k})=\frac{K_{\frac{\nu}{2}}\left(\sqrt{\alpha^{2}+k^{2}}\right)}{K_{\frac{\nu}{2}}(\alpha)} \frac{\left(\alpha^{2}+k^{2}\right)^{\frac{\nu}{4}}}{\alpha^{\frac{\nu}{2}}} .
$$

Hence the eventual result $\tilde{f}_{n}(\mathbf{k})=\tilde{f}_{1}(k)$.

\section{References}

1. R. Cont. Empirical properties of asset returns: stylized facts and statistical issues. Quantitative Finance, 1(2):223236, February 2001.

2. J. P. Bouchaud and M. Potters. Theory of financial risk and derivative pricing : from statistical physics to risk management. Cambridge Univ. Press, second edition, 2003.

3. T. Bollerslev, R. F. Engle, and D. B. Nelson. ARCH Models. In R. F. Engle and D. L. McFadden, editors, Handbook of Econometrics, pages 2959-3038. Elsevier, 1994.

4. R. S. Tsay. Analysis of Financial Time Series, chapter 3. John Wiley \& Sons, 2002.

5. M. Musiela and M. Rutkowski. Martingale Methods in Financial Modelling, chapter 7, pages 237-278. Springer Verlag, second edition, 2005.

6. B. Mandelbrot, A. Fisher, and L. Calvet. A multifractal model of asset returns. Cowles Foundation Discussion Papers 1164, Cowles Foundation, Yale University, September 1997.

7. E. Bacry, J. Delour, and J. F. Muzy. Modelling financial time series using multifractal random walks. Physica A, 299(1-2):84-92, 2001.

8. Z. Eisler and J. Kertész. Multifractal model of asset returns with leverage effect. Physica A, 343:603-622, November 2004 .
9. L. Borland, J. P. Bouchaud, J. F. Muzy, and G. O. Zumbach. The Dynamics of Financial Markets - Mandelbrot's multifractal cascades, and beyond. Science \& Finance (CFM) working paper archive 500061, Science \& Finance, Capital Fund Management, January 2005.

10. G. O. Zumbach, M. M. Dacorogna, J. L. Olsen, and R. B. Olsen. Measuring shock in financial markets. International Journal of Theoretical and Applied Finance, 3:347$355,2000$.

11. G. O. Zumbach. Volatility processes and volatility forecast with long memory. Quantitative Finance, 4(1):70-86, 2004.

12. L. Borland and J. P. Bouchaud. On a multi-timescale statistical feedback model for volatility fluctuations. Science \& Finance (CFM) working paper archive 500059, Science \& Finance, Capital Fund Management, July 2005.

13. R. Cont and P. Tankov. Financial Modelling with Jump Processes, chapter 4. Financial Mathematics Series. CRC Press, 2004.

14. P. Carr, H. Geman, D. Madan, and M. Yor. Selfdecomposability and option pricing. Mathematical finance, 17(1):31-57, 2007.

15. F. Baldovin and A. L. Stella. Central limit theorem for anomalous scaling due to correlations. Physical Review E, $75(2): 020101,2007$.

16. F. Baldovin and A. L. Stella. Scaling and efficiency determine the irreversible evolution of a market. Proc. Natl. Acad. Sci. USA, 104(50):19741-4, 2007.

17. F. Baldovin and A. L. Stella. Role of scaling in the statistical modeling of finance, 2008. Based on the Key Note lecture by A.L. Stella at the Conference on "Statistical Physics Approaches to Multi-Disciplinary Problems", IIT Guwahati, India, 7-13 January 2008. 
18. T. di Matteo. Multiscaling in finance. Qu. Fin., (1):21-36, 2007.

19. A. Andreoli, F. Caravenna, P.D. Pra, and G. Posta. Scaling and multiscaling in financial indexes: a simple model. Arxiv preprint arXiv:1006.0155, 2010.

20. I. M. Sokolov, A. V. Chechkin, and J. Klafter. Fractional diffusion equation for a power-law-truncated Lévy process. Physica A, 336(3-4):245-251, May 2004.

21. R. Osorio, L. Borland, and C. Tsallis. Distributions of highfrequency stock market observables. In M. Gell-Mann and C. Tsallis, editors, Nonextensive entropy: interdisciplinary applications, page 321. Oxford University Press, 2004

22. R Development Core Team. R: A Language and Environment for Statistical Computing. R Foundation for Statistical Computing, Vienna, Austria, 2008.

23. C. Vignat and A. Plastino. Scale invariance and related properties of $q$-Gaussian systems. Physics Letters $A, 365: 370-375$, June 2007.

24. E. Eberlein and U. Keller. Hyperbolic distributions in finance. Bernoulli, 1(3):281-299, 1995.

25. N. H. Bingham and R. Kiesel. Modelling asset returns with hyperbolic distributions. In J. Knight and S. Satchell, editors, Return Distributions in Finance, chapter 1, pages 1-20. Butterworth-Heinemann, 2001.

26. R. B. Nelsen. An introduction to copulas. Springer Series in Statistics. Springer, second edition, 2006.

27. U. Cherubini, E. Luciano, and W. Vecchiato. Copula methods in finance. Wiley Finance. Wiley, 2004.

28. Y. Malevergne and D. Sornette. Extreme Financial Risks. Springer, 2006.

29. I.J. Schoenberg. Positive definite functions on spheres. Duke Math. Journal, 9:96-108, 1942.
30. N. H. Bingham and R. Kiesel. Semi-parametric modelling in finance: theoretical foundations. Quantitative Finance, 1:1-10, 2001.

31. F. Lillo and R. N. Mantegna. Power-law relaxation in a complex system: Omori law after a financial market crash. Physical Review E, 68(1):016119, Jul 2003.

32. P. Weber, F. Wang, I. Vodenska-Chitkushev, S. Havlin, and H. E. Stanley. Relation between volatility correlations in financial markets and Omori processes occurring on all scales. Physical Review E, 76(1):016109, 2007.

33. F. Lillo. Limit order placement as an utility maximization problem and the origin of power law distribution of limit order prices. European Physical Journal B, 55:453-459, February 2007.

34. J. L. McCauley, K. E. Bassler, and G. H. Gunaratne. Martingales, the efficient market hypothesis, and spurious stylized facts, October 2007.

35. Raffaello Morales, T. Di Matteo, Ruggero Gramatica, and Tomaso Aste. Dynamical generalized hurst exponent as a tool to monitor unstable periods in financial time series. Physica A, (391):3180-3189, 2012.

36. R. H. Riedi. Multifractal processes. In P. Doukhan, G. Oppenheim, and M. S. Taqqu, editors, Long-range Dependence: Theory and Applications, pages 625-716. Birkhauser, 2002.

37. S. Jaffard. Multifractal Formalism for Functions Part I: Results Valid for All Functions. SIAM Journal on Mathematical Analysis, 28:944-970, 1997.

38. S. Jaffard. Multifractal Formalism for Functions Part II: Self-Similar Functions. SIAM Journal on Mathematical Analysis, 28:971-998, 1997. 
39. E. Bacry, J. Delour, and J. F. Muzy. Multifractal random walk. Physical Review E, 64(2):26103, 2001.

40. E. Bacry, A. Kozhemyak, and J. F. Muzy. Are asset return tail estimations related to volatility long-range correlations? Physica A, 370(1):119-126, Oct 2006.

41. J. F. Muzy, E. Bacry, and A. Kozhemyak. Extreme values and fat tails of multifractal fluctuations. Physical Review E, 73(6):066114, 2006.

42. J. P. Bouchaud. Elements for a theory of financial risks. Physica A, 263:415-426, February 1999.

43. J. P. Bouchaud, M. Potters, and M. Meyer. Apparent multifractality in financial time series. European Physical Journal B, 13:595-599, January 2000.

44. Z. Q. Jiang and W. X. Zhou. Multifractality in stock indexes: Fact or fiction? Physica A, 387:3605-3614, June 2008.

45. D. Sornette. Critical market crashes. Physics Reports, 378(1):1-98, 2003.

46. D. Sornette, Y. Malevergne, and J. F. Muzy. Volatility fingerprints of large shocks: Endogeneous versus exogeneous. The Journal of Risk, 16(2):67-71, February 2003.

47. G. Chang and J. Feigenbaum. A bayesian analysis of logperiodic precursors to financial crashes. Quantitative Finance, 6:15-36, 2006.

48. A. Helmstetter, D. Sornette, and J. R. Grasso. Mainshocks are aftershocks of conditional foreshocks: How do foreshock statistical properties emerge from aftershock laws. Journal of Geophysical Research, 108:2046, 2003.

49. J. P. Bouchaud, A. Matacz, and M. Potters. Leverage effect in financial markets: The retarded volatility model. Physical Review Letters, 87(22):228701, Nov 2001.
50. P. E. Lynch and G. O. Zumbach. Market heterogeneities and the causal structure of volatility. Quantitative $F i$ nance, 3(4):320-331, 2003.

51. G. O. Zumbach. Time reversal invariance in finance, August 2007 .

52. S. Drozdz, M. Forczek, J. Kwapien, P. Oswiecimka, and R. Rak. Stock market return distributions: From past to present. Physica A, 383(1):59-64, Sep 2007.

53. C. C. Heyde and N. N. Leonenko. Student processes. Advances in Applied Probability, 37:342-365, 2005.

54. L. Borland. Option pricing formulas based on a nongaussian stock price model. Physical Review Letters, 89(9):98701, 2002.

55. M. Vellekoop and H. Nieuwenhuis. On option pricing models in the presence of heavy tails. Quantitative Finance, 7(5):563-573, Oct 2007.

56. O.E. Barndorff-Nielsen and N. Shephard. Non-Gaussian Ornstein-Uhlenbeck-based models and some of their uses in financial economics. Journal of the Royal Statistical Society: B, 63(2):167-241, 2001.

57. A. Erdélyi. Higher Transcendental Functions (Vol. 2). McGraw-Hill Publisher, 1953.

58. S. Hurst. The characteristic function of the Student $t$ distribution. Technical Report SRR95-044, Austrialian National University, Centre for Mathematics and its Applications, Canberra, September 1995.

59. I. Dreier and S. Kotz. A note on the characteristic function of the t-distribution. Statistics \& Probability Letters, 57(3):221-224, 2002. 